\title{
Comparison Among HB-inspired Algorithms for Continuous-Spin Systems and Gauge Fields
}

\author{
A. Cucchieri, R. B. Frigori, T. Mendes, and A. Mihara \\ Instituto de Física de São Carlos - Universidade de São Paulo \\ C.P. 369, 13560-970, São Carlos, SP, Brazil
}

Received on 30 September, 2005

\begin{abstract}
We propose a new local algorithm for the thermalization of $n$-vector spin models, which can also be used in the numerical simulation of $S U(N)$ lattice gauge theories. The algorithm combines heat-bath (HB) and microcanonical updates in a single step - as opposed to the hybrid overrelaxation method, which alternates between the two kinds of update steps - while preserving ergodicity. We test our proposed algorithm in the case of the one-dimensional 4-vector spin model and compare its performance with the standard HB algorithm and with other HB-inspired algorithms.
\end{abstract}

Keywords: Monte Carlo algorithms, Heat-bath; Overrelaxation; Critical slowing-down

\section{INTRODUCTION}

The lattice formulation of quantum field theories provides a first principles approach to investigate non-perturbative aspects of high-energy physics. In this formulation the theory becomes equivalent to a statistical mechanical model, which can be studied numerically using Monte Carlo simulations (see for example [1,2] and references therein). As a consequence, the system considered evolves according to a Markov process in the so-called Monte Carlo time and the actionweighted configuration-space average of the observables is substituted by a time average over successive (independent) field configurations of the system. The possibility to use Monte Carlo simulations to study the theory nonperturbatively is especially important in the case of quantum chromodynamics, the nonabelian $S U(3)$ gauge theory describing the strong interactions between hadrons. These simulations are computationally very demanding and must be done using local thermalization algorithms, since global methods (such as cluster algorithms) do not work well in this case.

We should notice that, based on the above-mentioned equivalence, when the continuum limit of the lattice quantum field theory is taken, the corresponding statistical mechanical model approaches its critical point. More precisely, the distance from the critical point is given by the lattice parameter $\beta$ (directly related to the lattice bare coupling constant), which corresponds to an inverse temperature. For nonabelian gauge theories (as a consequence of asymptotic freedom) the continuum limit is given by $\beta \rightarrow \infty$, i.e. the critical point corresponds to temperature zero. The expected critical behavior is therefore similar to the one of a two-dimensional continuous-spin model (e.g. the classical Heisenberg or $n$-vector spin model with $n>2$ ) or of a one-dimensional spin model, which show a critical point only at temperature zero, or $\beta \rightarrow \infty$.

The process of obtaining independent field configurations is called thermalization and is usually carried out by applying at each link of the lattice a local algorithm, such as Metropolis or heat bath (HB). When a critical point is approached, this process is afflicted by the well-known phenomenon of critical slowing-down (CSD) [3], which increases the correlation among successive field configurations. This implies that the integrated auto-correlation time $\tau_{\text {int }}$ increases as a power of the lattice side $N$. In particular, for the Metropolis or HB algorithms one has $\tau_{\text {int }} \sim N^{2}$, i.e. the dynamic critical exponent $z$ is equal to 2. Since statistical Monte-Carlo errors are proportional to $\sqrt{2 \tau_{\text {int }}}$, numerical simulations become increasingly inefficient close to a critical point. In order to reduce the problem of CSD one can combine the standard Metroplis and $\mathrm{HB}$ algorithms with so-called micro-canonical updates, allowing larger jumps in the configuration space and therefore improving the generation of independent samples. This is the idea behind the so-called hybrid overrelaxation method [4]. In general, adding a few micro-canonical sweeps greatly reduces CSD and, correspondly, the computational work.

A modification of the heat-bath algorithm, called overheatbath (OHB), was introduced some years ago in Ref. [5]. The basic idea was to incorporate a micro-canonical move directly into the heat-bath step, thus reducing the computational cost while preserving the large moves in the configuration space. As it turns out, combining the two moves (heat-bath and micro-canonical) in a single step leads to a significant improvement in performance when compared to the hybrid overrelaxation method described above, which is based on alternating the two kinds of update moves. The resulting algorithm was indeed able to speed up the thermalization process, but, as already stressed in Ref. [5], it is not clear if it preserves ergodicity (especially when working at small temperatures). The OHB algorithm is used today in numerical simulations [6], usually combined with other algorithms in order to ensure ergodicity. In this work we propose a modification of the overheat-bath algorithm, which we call the modified heat-bath algorithm (MHB), incorporating a micro-canonical move into the heat-bath step without compromising ergodicity. In order to test the MHB algorithm - and compare its performance with the standard HB algorithm and the OHB algorithm we consider the 4-vector spin model on a 1-dimensional lattice. Note that the model is exactly solvable, which makes it especially suited for testing the algorithm and comparing results to the exact solution.

Let us also note that, due to the isometry between the groups $O(4)$ and $S U(2)$, it is possible to study the $S U(2)$ case 
with the same algorithm used for the 4-vector case. More precisely, the local update - i.e. the update of a single spin or gauge field variable while holding the rest of the lattice fixed - of the $S U$ (2) lattice gauge theory is identical to the one for the 4-vector model. This does not mean, however, that the global update of an $S U(2)$ gauge field configuration can be obtained from the corresponding update of a 4-vector model. Indeed, the latter update can be done very efficiently using the Swendsen-Wang-Wolff algorithm, while no such class of algorithms works well for lattice gauge theories. The method proposed here is therefore intended mostly for use in simulations of lattice gauge theories and not of $n$-vector models, although hybrid overrelaxation methods have also been applied in large-scale simulations of $n$-vector models [7]. Of course, an efficient thermalization in the $S U(2)$ case is of great physical interest, since the quenched $S U\left(N_{c}\right)$ case (for $N_{c} \geq 3$ ) is usually studied applying the $S U(2)$-embedding technique introduced in Ref. [8]. The $S U(2)$ embedding is also used for simulating other Lie groups, such as the $S p(2)$ and $S p(3)$ groups, in studies of the deconfinement phase transition [9]. Preliminary results for the $2 d S U(2)$ case have been presented in [10].

\section{THE 4-VECTOR SPIN MODEL AND THE ALGORITHMS}

The 4-vector spin model (on a 1-d lattice) is defined by the Hamiltonian

$$
\mathcal{H}=-\beta \sum_{x=1}^{N} S_{x} \cdot S_{x+1},
$$

where the spins $S_{x}$ are four-dimensional unit vectors, $\beta \sim 1 /$ Temperature, $N$ is the lattice side and . indicates a scalar product.

In the case of a local algorithm, one has to consider the contribution to the Hamiltonian due to a single spin $S_{x}$. This gives $\mathcal{H}_{s s}=-\beta S_{x} \cdot H_{x}+$ constant, where the "effective magnetic field" $H_{x}$ is given by $H_{x}=S_{x-1}+S_{x+1}$. (Here we consider periodic boundary conditions.) The above equation can also be written as

$$
\mathcal{H}_{s s}=-\frac{\beta N_{x}}{2} \operatorname{Tr} S_{x} \widetilde{H}_{x}^{\dagger}+\text { constant },
$$

where $S_{x}$ and $\widetilde{H}_{x}$ are now interpreted as $S U(2)$ matrices in the fundamental representation and $N_{x}=\sqrt{\operatorname{det} H_{x}}$. Note that Eq. (2) is clearly analogous to the expression of the single-link action obtained by considering the contribution of a single link variable to the $S U(2)$ Wilson action [10].

Using the single-side action (2) and the invariance of the group measure under group multiplication, one obtains the HB update [11]

$$
S_{x}^{\text {old }} \rightarrow S_{x}^{\text {new }}=U \widetilde{H}_{x}
$$

Here, $U=u_{0} \mathrm{I}+i \sum_{j} u_{j} \sigma_{j}$ is an $S U(2)$ matrix, $I$ is the $2 \times$ 2 identity matrix, $\sigma_{j}$ are the three Pauli matrices and $u_{0}$ is randomly generated according to the distribution

$$
\sqrt{1-u_{0}^{2}} \exp \left(\beta N_{x} u_{0}\right) d u_{0} \text {. }
$$

At the same time, the vector $\vec{u}$ - with components $u_{j}$ normalized to $\sqrt{1-u_{0}^{2}}$ - points along a uniformly chosen random direction in three-dimensional space [2].

In the hybrid over-relaxed algorithm one does $m$ microcanonical (or energy-conserving) update sweeps over the lattice, followed by one HB sweep. The micro-canonical update is a local deterministic transformation applied to the $S U(2)$ matrix $S_{x}$, which does not change the value of the Hamiltonian. This is achieved by considering the update

$$
S_{x}^{\text {old }} \rightarrow S_{x}^{\text {new }}=\widetilde{H}_{x} \operatorname{Tr}\left[S_{x}^{\text {old }} \widetilde{H}_{x}^{\dagger}\right]-S_{x}^{\text {old }} .
$$

As stressed in the Introduction, this update represents a large move in the configuration space, allowing the hybrid overrelaxed algorithm to reduce CSD at the price of a greater computational cost, due to the micro-canonical sweeps. Usually, by setting $m=2,3$ one obtains a strong reduction in the value of $\tau_{\text {int }}$ while increasing the computational cost by a factor smaller than 2 .

In the OHB [5] one tries to include the micro-canonical step (5) directly into the heat-bath algorithm. To this end one generates $u_{0}$ according to the distribution (4) while the components of $\vec{u}$ are not randomly chosen but are set using the relation $\vec{u}=-\vec{w}$, where $W=w_{0} \mathrm{I}+i \vec{\sigma} \cdot \vec{w}=S_{x}^{\text {old }} \widetilde{H}_{x}^{\dagger}$. As a final step, the vector $\vec{u}$ is normalized to $\sqrt{1-u_{0}^{2}}$. Clearly, the idea here is the same one applied in the standard hybrid over-relaxed algorithm: one tries to maximize the move in the configuration space by changing the sign of the component of $W$ that is orthogonal to the effective magnetic field. (Note that the action $S=-\beta N / 2 \operatorname{Tr} W$ can be viewed as the action of a matrix $W$ in an effective magnetic field given by the identity matrix I.) Clearly, this step does not obey the uniform distribution for $\vec{u}$ but is a microcanonical move. Indeed we can think of the algorithm as a two-step process: a HB move followed by a microcanonical step. Thus, the algorithm is exact but may not be ergodic. We analyze the conditions for applicability of the OHB algorithm in a separate work [12], but it is clear that for some initial configurations the algorithm can get "trapped" inside a subset of the space of configurations, compromising the ergodicity condition. One such configuration is, for example, a "cold" start for an $n$-vector model, in which all spins are aligned along a fixed direction. In this case, the OHB is not able to change the lattice configuration at all. There is a clear problem also if the initial configuration in the $S U(2)$-lattice-gauge-theory case is given by variables in an Abelian subgroup. As can be easily seen, the update will change the initial configuration in this case, but the resulting Markov chain will remain restricted to the Abelian sector, without exploring the full space of configurations.

In this work we propose a modified $\mathrm{HB}$ algorithm (MHB) in which the generation of the $S U(2)$ matrix $U$ is done as in the $\mathrm{HB}$ case, but followed by one final step: if the scalar product $\vec{u} \cdot \vec{w}$ is positive then one does $\vec{u} \rightarrow-\vec{u}$, where the matrix $W$ 

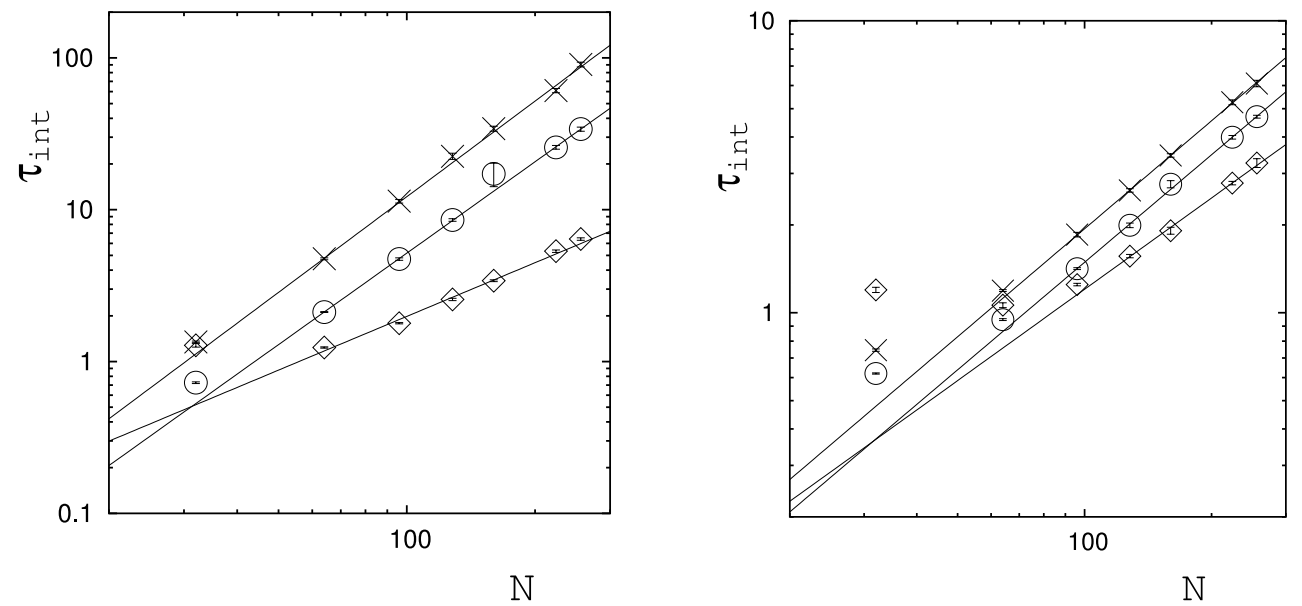

FIG. 1: Integrated auto-correlation time of the susceptibility as a function of the lattice side $N$ for $\mathrm{HB}(\times)$, OHB ( $\diamond)$ and MHB (०), with 0 (left) and 1 (right) overrelaxation step. Data have been fitted to the Ansatz $\tau_{i n t}=a N^{z}$.
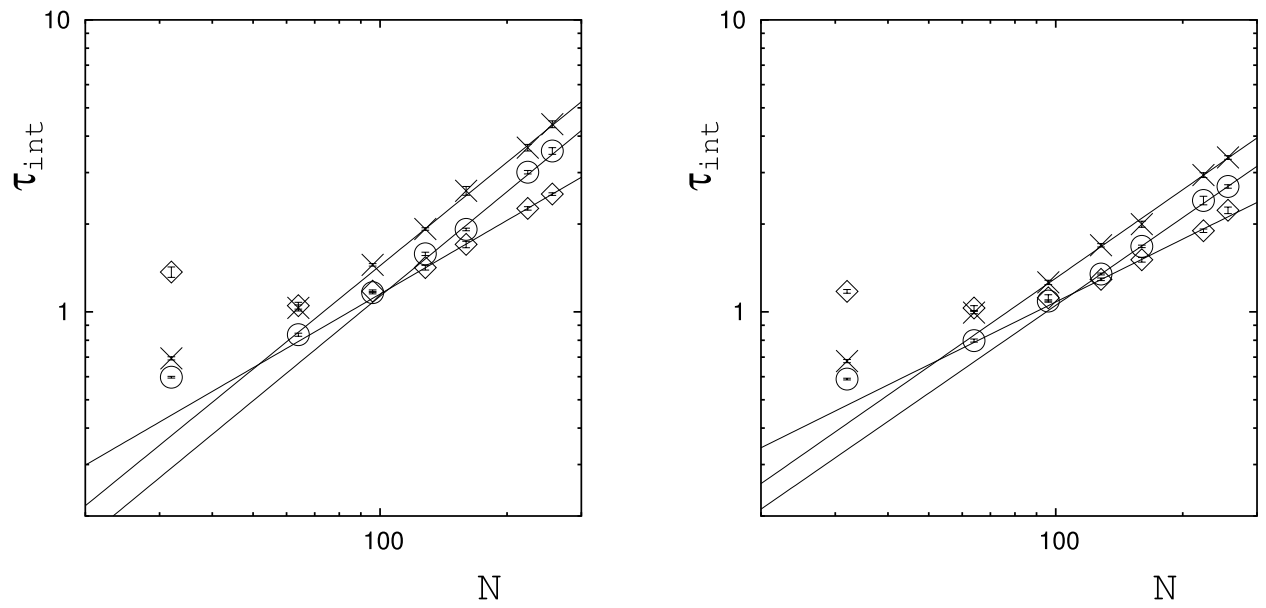

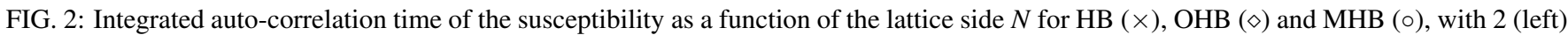
and 3 (right) overrelaxation step. Data have been fitted to the Ansatz $\tau_{i n t}=a N^{z}$.

has been defined above. This may also be thought of as a modification of the overheat-bath (OHB). As said above, the basic idea, in both cases, is to incorporate a micro-canonical move into the heat-bath step. The difference is that in our case the direction of $\vec{u}$ is randomly chosen and only its sign is modified, according to the above rule, while in the OHB algorithm one fixes $\vec{u} \propto-\vec{w}$ directly. Our modification should ensure ergodicity while keeping the advantage of having a micro-canonical step included into the HB step. We have indeed verified that the MHB algorithm has no problem with cold starts and shows a better $\tau_{\text {int }}$ than that of the OHB algorithm for energy-related observables. On the other hand, in the OHB case, the move in configuration space is more optimized and the iteration cost is lower, since the algorithm is simpler and it needs fewer random numbers. Our results are presented in the next section.

\section{RESULTS AND CONCLUSIONS}

In order to study the CSD of the new algorithm we have to investigate if, and with what exponent, its relaxation time $\tau_{\text {int }}$ (for certain quantities we are interested in) diverges as the lattice size $N$ increases. To this end, we have to evaluate $\tau_{\text {int }}$ for different lattice sides $N$ at "constant physics", namely as the lattice size is increased, the physical size of the lattice should remain fixed. This is done by introducing a correlation length $\xi$ and by keeping the ratio $\xi / N$ fixed, with $N \ll \xi$ in order to keep finite-size effects under control. For the 1d 4-vector spin model one has $\xi \propto \beta$ [13], thus one should tune $N \propto \beta$ in order to keep the ratio $\xi / N$ constant. In our simulations we considered the lattice sizes $N=32,64,96$, $128,160,224,256$ and $\beta=2.5 N / 32$ and $\beta=5.0 N / 32$. In all cases we did $1.1 \times 10^{6}$ thermalization sweeps and for the 
analysis we discarded the first $10^{5}$ sweeps.

In order to test the new algorithm we have evaluated the energy density $\varepsilon=N^{-1} \sum_{x=1}^{N} S_{x} \cdot S_{x+1}$, the magnetization $\mathcal{M}^{2}=$ $\left(\sum_{x=1}^{N} S_{x}\right)^{2}$ and the magnetic susceptibility $\chi=N^{-1}\left\langle\mathcal{M}^{2}\right\rangle$. Let us recall that there exists an exact solution [13] for the 1d 4-vector spin model, even at finite lattice side $N$ and with periodic boundary conditions. Thus, we can check the numerical results for the various quantities against the exact solution (see also [14, Table 1]).

In all cases we have evaluated the integrated autocorrelation time $\tau_{\text {int }}$ using an automatic windowing procedure [3] with two different window factors ( $c=4$ and 8$)$. We also employ a method based on a comparison between the naive statistical error with a binning error [15]. We checked that these three estimates are always in agreement. The error on the integrated auto-correlation time $\tau_{\text {int }}$ has been evaluated using the Madras-Sokal formula [3].

Results are reported in Figs. 1 and 2. The data obtained for $\tau_{\text {int }}$ have been fitted to the Ansatz $\tau_{\text {int }}=a N^{z}$ in order to estimate the dynamic critical exponent $z$. Our study shows that the MHB algorithm has essentially the same critical exponent $z$ of the standard HB algorithm but the value of the integrated auto-correlation time $\tau_{\text {int }}$ is about $30 \%$ smaller compared to the standard HB algorithm. This implies a reduction in the statistical error and in the computational cost by a factor of about $20 \%$. Similar results have been obtained in the $S U(2)$ case [10]. Thus, a good decorrelation can be reached without the use of multiple micro-canonical sweeps. In particular, from our simulations it appears that the HB algorithm with $m$ micro-canonical steps is essentially equivalent to the MHB algorithm with $m-1$ micro-canonical steps. This feature may be useful in parallel computing [2] due to the reduction of the inter-node communication.

At the same time, from our data one sees that the MHB algorithm has a critical exponent $z$ larger than that of the $\mathrm{OHB}$ algorithm. In order to reduce CSD for the MHB algorithm, while keeping its ergodicity properties, we are now testing an algorithm that interpolates between MHB and OHB.

A more extensive study of these algorithms, both in the $O(4)$ and in the $S U(2)$ cases, will be presented in a forthcoming work [12].

\section{Acknowledgments}

Research supported by FAPESP (Projects No. 00/05047-5 and 03/00928-1). Partial support from CNPq is also acknowledged (AC, TM).
[1] T. Mendes, "Computational aspects of lattice QCD", arXiv:heplat/0309127.

[2] A. Cucchieri, T. Mendes, G. Travieso, and A. R. Taurines, arXiv:hep-lat/0308005.

[3] A. D. Sokal, "Monte Carlo methods in statistical mechanics: foundations and new algorithms", http://citeseer.nj.nec.com/sokal96monte.html , (1996).

[4] U. Wolff, Phys. Lett. B 288, 166 (1992).

[5] R. Petronzio and E. Vicari, Phys. Lett. B 254, 444 (1991).

[6] R. Fiore, A. Papa, and P. Provero, Phys. Rev. D 67, 114508 (2003).

[7] K. Chen and D.P. Landau, Phys. Rev. B 49, 3266 (1994).

[8] N. Cabibbo and E. Marinari, Phys. Lett. B 119, 387 (1982).
[9] K. Holland, M. Pepe, and U. J. Wiese, Nucl. Phys. B 694, 35 (2004).

[10] R. B. Frigori, A. Cucchieri, T. Mendes, and A. Mihara, AIP Conf. Proc. 739, 593 (2005).

[11] M. Creutz, Phys. Rev. D 21, 2308 (1980).

[12] A. Cucchieri, R. B. Frigori, T. Mendes, and A. Mihara, "Improved heat-bath dynamics for continuous-spin systems and gauge theories", in preparation.

[13] A. Cucchieri, T. Mendes, A. Pelissetto, and A. D. Sokal, J. Statist. Phys. 86, 581 (1997).

[14] T. Mendes and A. D. Sokal, Phys. Rev. D 53, 3438 (1996).

[15] G. de Divitiis et al., Nucl. Phys. B 437, 447 (1995). 